\title{
Simulated solar light irradiation of mesotrione in
}

\section{natural waters}

\author{
Alexandra ter Halle and Claire Richard* \\ Laboratoire de Photochimie Moléculaire et Macromoléculaire, UMR n6505 CNRS-Université \\ Blaise Pascal, 63177 Aubière Cedex, France
}

This supporting information contains the details of the experiments. 


\section{Supporting Informations}

\section{Irradiation using "black light" lamps}

The mesotrione (or model compound) concentration in solution was monitored by HPLC analysis during the irradiation experiments. For each experiment the initial solution was injected three times. After irradiation the sample were injected two times. The results presented here were obtained by averaging the measured HPLC area. The standard error for every point is majored at $2 \%$.

The model compounds used in this study are phenol (P), 2,4,6-trimethylphenol (TMP) and coniferyl alcohol (CA).

Experiment 1

Irradiation of a solution of mesotrione (Mes) in aerated medium $(0.1 \mathrm{mM})$ in $\mathrm{pH} 6.5$ buffered water. Experiment 2

Irradiation of a solution of mesotrione in deoxygenated medium $(0.1 \mathrm{mM})$ in $\mathrm{pH} 6.5$ buffered water.

\begin{tabular}{|c|c|c|c|c|c|c|c|c|c|c|c|}
\hline & $\begin{array}{c}\text { Irradiation } \\
\text { time }\end{array}$ & $0 \mathrm{~h}$ & $1 \mathrm{~h}$ & $1 \mathrm{~h} 30$ & $2 \mathrm{~h}$ & $2 \mathrm{~h} 50$ & $3 \mathrm{~h}$ & $5 \mathrm{~h}$ & $5 \mathrm{~h} 30$ & $7 \mathrm{~h}$ & $8 \mathrm{~h}$ \\
\hline 1 & $\begin{array}{c}\text { Concentration } \\
* 10^{5} \mathrm{~mol} / \mathrm{L} \\
\pm 0.1^{*} 10^{5} \\
\mathrm{~mol} / \mathrm{L}\end{array}$ & 10 & 9.3 & & 9.0 & 8.6 & & 8.0 & & 7.5 & 7.1 \\
\hline 2 & $\begin{array}{c}\text { Concentration } \\
* 10^{5} \mathrm{~mol} / \mathrm{L} \\
\pm 0.1^{*} 10^{5} \\
\mathrm{~mol} / \mathrm{L}\end{array}$ & 10 & & 8.1 & & & 6.6 & & 5.1 & 5 & \\
& & & & & & & & & & & \\
\hline
\end{tabular}

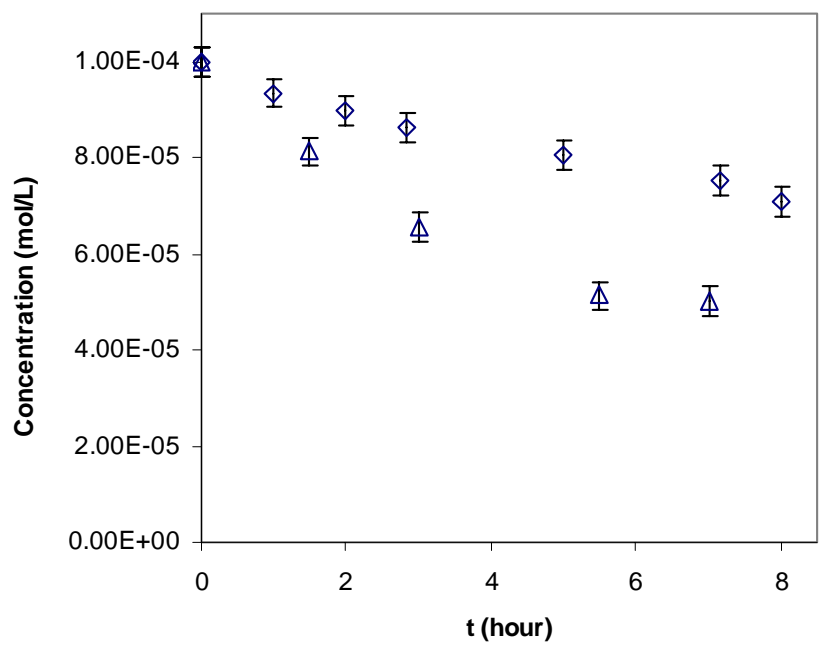

Concentration decay versus time for mesotrione $(\diamond)$ in aerated media and $(\Delta)$ in deoxygenated solution. The exponential fit decay is represented in dashed line.

\section{Experiment 3}

Irradiation of a solution of $\mathrm{P}(0.5 \mathrm{mM})$ in $\mathrm{pH} 6.5$ buffered water. 


\section{Experiment 4}

Irradiation of a solution of TMP $(0.5 \mathrm{mM})$ in $\mathrm{pH} 6.5$ buffered water.

Experiment 5

Irradiation of a solution of $\mathrm{CA}(0.5 \mathrm{mM})$ in $\mathrm{pH} 6.5$ buffered water.

\begin{tabular}{|c|c|c|c|c|c|c|c|c|c|}
\hline & $\begin{array}{c}\text { Irradiation } \\
\text { time }\end{array}$ & $0 \mathrm{~h}$ & $1 \mathrm{~h}$ & $2 \mathrm{~h}$ & $3 \mathrm{~h}$ & $5 \mathrm{~h}$ & $7 \mathrm{~h}$ & $10 \mathrm{~h}$ & $14 \mathrm{~h}$ \\
\hline 3 & $\begin{array}{c}\text { Concentration } * 10^{5} \\
\mathrm{~mol} / \mathrm{L} \\
\pm 0.1 * 10^{5} \mathrm{~mol} / \mathrm{L}\end{array}$ & 50 & & & 50 & & 50 & & \\
\hline 4 & $\begin{array}{c}\text { Concentration } * 10^{5} \\
\mathrm{~mol} / \mathrm{L} \\
\pm 0.1 * 10^{5} \mathrm{~mol} / \mathrm{L}\end{array}$ & 50 & 49 & 50 & 49.5 & 50 & 49.5 & 50 & 48.5 \\
\hline 5 & $\begin{array}{c}\text { Concentration } * 10^{5} \\
\mathrm{~mol} / \mathrm{L} \\
\pm 0.1 * 10^{5} \mathrm{~mol} / \mathrm{L}\end{array}$ & 50 & & 45 & 43.5 & 36.5 & 32.5 & & \\
\hline
\end{tabular}

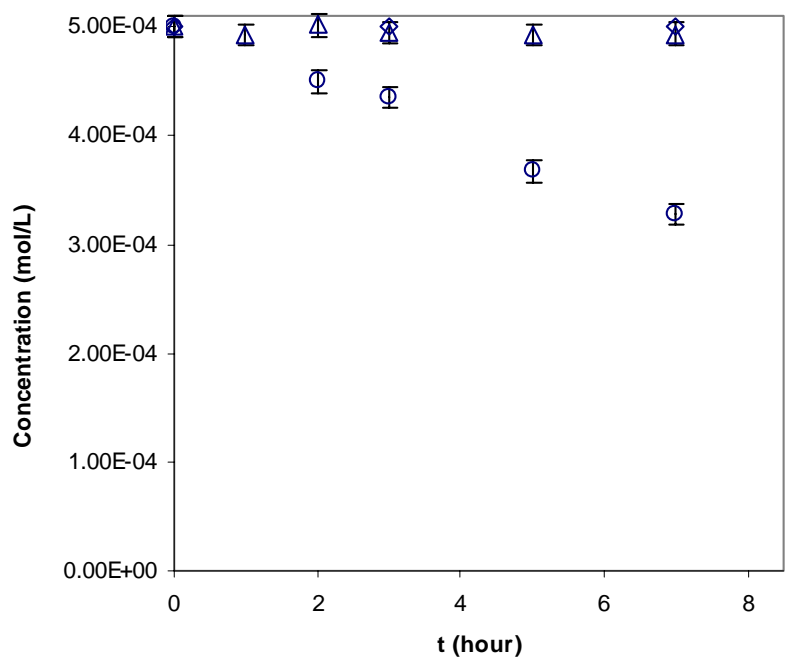

Concentration decay versus time $(\Delta)$ for TMP $(\diamond)$ for Phenol and $(\mathrm{O})$ for CA

\section{Experiment 6}

Irradiation of a solution of Mes $(0.1 \mathrm{mM})$ and $\mathrm{P}(0.5 \mathrm{mM})$ in $\mathrm{pH} 6.5$ buffered water.

\begin{tabular}{|c|c|c|c|c|}
\hline $\begin{array}{c}\text { Irradiation } \\
\text { time }\end{array}$ & $0 \mathrm{~h}$ & $2 \mathrm{~h}$ & $5 \mathrm{~h}$ & $7 \mathrm{~h} 45$ \\
\hline $\begin{array}{c}\text { Mes concentration } \\
\left(* 10^{5} \mathrm{~mol} / \mathrm{L}\right) \\
\pm 0.1 * 10^{5} \mathrm{~mol} / \mathrm{L}\end{array}$ & 10 & 10 & 9.7 & 9.8 \\
\hline $\begin{array}{c}\mathrm{P} \text { concentration } \\
\left(* 10^{5} \mathrm{~mol} / \mathrm{L}\right) \\
\pm 0.1 * 10^{5} \mathrm{~mol} / \mathrm{L}\end{array}$ & 50 & 48.6 & 47.8 & 46.3 \\
\hline
\end{tabular}




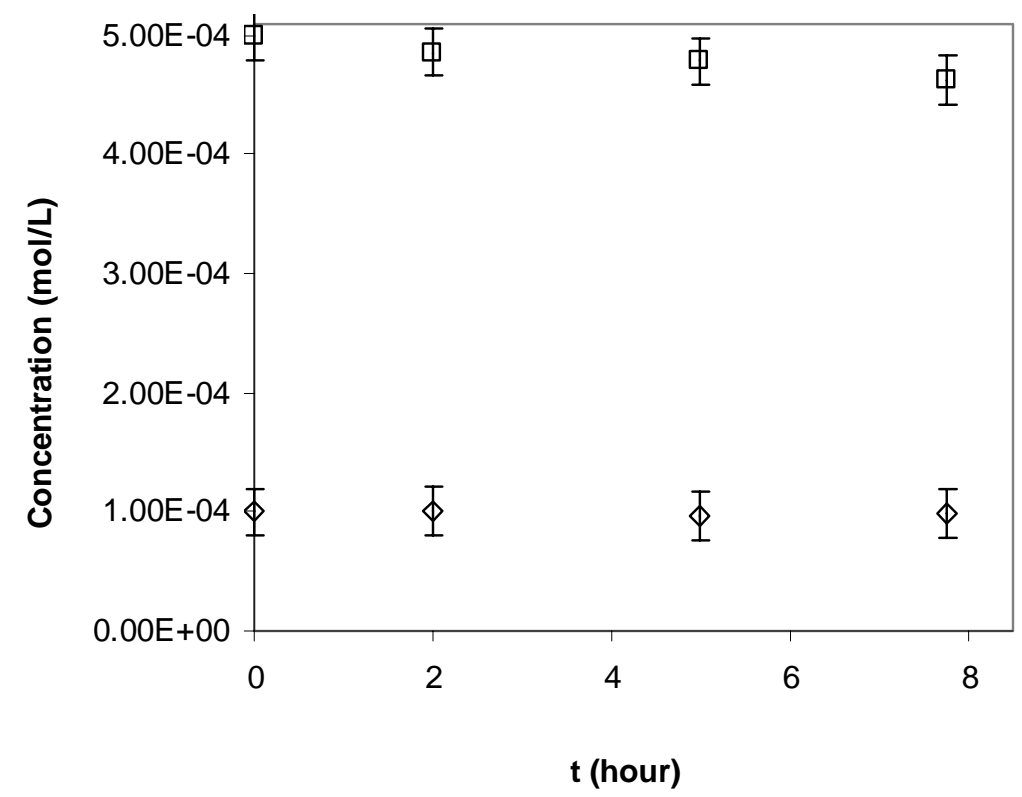

Concentration decay versus time for $(\diamond)$ Mes and $(\Delta) \mathrm{P}$

\section{Experiment 7}

Irradiation of a solution of Mes $(0.1 \mathrm{mM})$ and TMP $(0.5 \mathrm{mM})$ in $\mathrm{pH} 6.5$ buffered water.

\begin{tabular}{|c|c|c|c|c|c|c|c|c|c|}
\hline $\begin{array}{c}\text { Irradiation } \\
\text { time }\end{array}$ & $0 \mathrm{~h}$ & $\begin{array}{c}30 \\
\mathrm{~min}\end{array}$ & $1 \mathrm{~h}$ & $1 \mathrm{~h} 30$ & $2 \mathrm{~h}$ & $2 \mathrm{~h} 45$ & $3 \mathrm{~h}$ & $3 \mathrm{~h} 30$ & $4 \mathrm{~h}$ \\
\hline $\begin{array}{c}\text { Mes concentration } \\
\left(* 10^{5} \mathrm{~mol} / \mathrm{L}\right) \\
\pm 0.1 * 10^{5} \mathrm{~mol} / \mathrm{L}\end{array}$ & 10 & 9.9 & 10 & 9.8 & 10 & 9.8 & 9.9 & 10 & 9.9 \\
\hline $\begin{array}{c}\text { TMP concentration } \\
\left(* 10^{5} \mathrm{~mol} / \mathrm{L}\right) \\
\pm 0.1 * 10^{5} \mathrm{~mol} / \mathrm{L}\end{array}$ & 50 & 47 & 46 & 43 & 42 & 39 & 38 & 37 & 34.6 \\
\hline
\end{tabular}

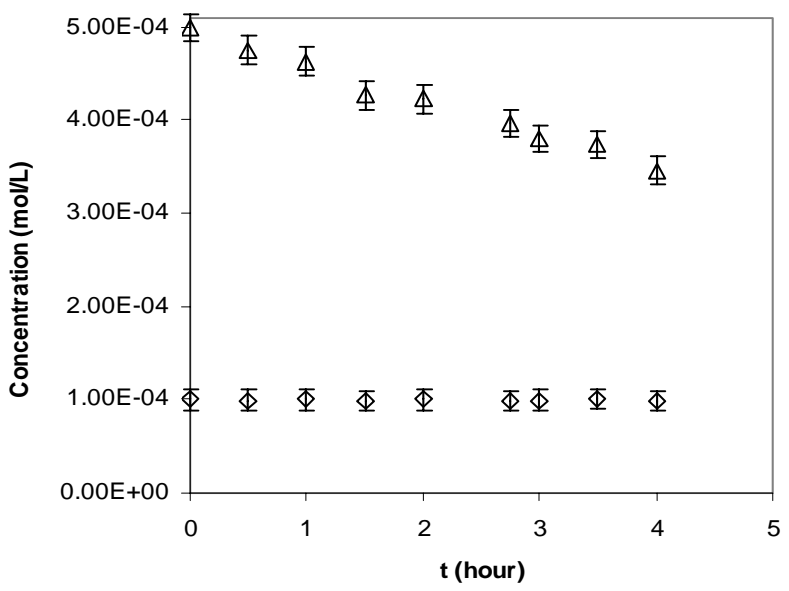

Concentration decay versus time for $(\diamond)$ Mes and $(\Delta)$ TMP 


\section{Experiment 8}

Irradiation of a solution of Mes $(0.1 \mathrm{mM})$ and CA $(0.5 \mathrm{mM})$ in $\mathrm{pH} 6.5$ buffered water.

\begin{tabular}{|c|c|c|c|c|c|c|}
\hline $\begin{array}{c}\text { Irradiation } \\
\text { time }\end{array}$ & $0 \mathrm{~h}$ & $1 \mathrm{~h}$ & $2 \mathrm{~h}$ & $3 \mathrm{~h}$ & $5 \mathrm{~h}$ & $7 \mathrm{~h}$ \\
\hline $\begin{array}{c}\text { Mes concentration } \\
\left(* 10^{5} \mathrm{~mol} / \mathrm{L}\right) \\
\pm 0.1 * 10^{5} \mathrm{~mol} / \mathrm{L}\end{array}$ & 10 & 9.9 & 10 & 9.8 & 9.8 & 9.9 \\
\hline $\begin{array}{c}\mathrm{AC} \text { concentration } \\
\left(* 10^{5} \mathrm{~mol} / \mathrm{L}\right) \\
\pm 0.1 * 10^{5} \mathrm{~mol} / \mathrm{L}\end{array}$ & 50 & 43 & 35 & 28 & 20 & 15 \\
\hline
\end{tabular}

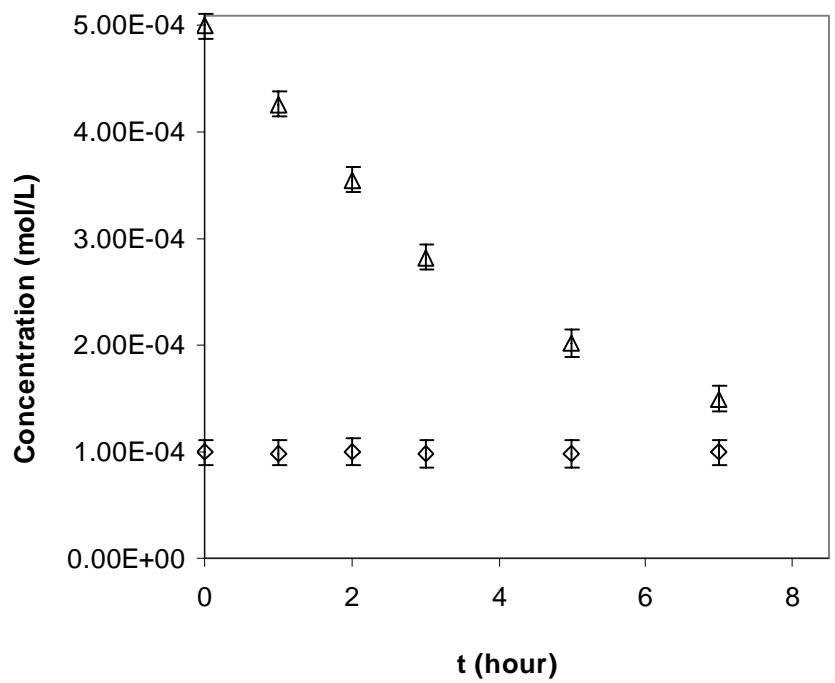

Concentration decay versus time for $(\diamond)$ Mes and $(\Delta)$ AC

\section{Irradiation using Suntest Photoreactor}

The percentage of mesotrione left in solution was measured by HPLC analysis. For each experiment the initial solution was injected five times. Each experiment was repeated twice and each aliquot was injected three times. The results presented are obtained by averaging of the collected data. The standard error for every point is majored at $2 \%$.

Experiment 9

Irradiation of a solution of mesotrione $(0.1 \mu \mathrm{M})$ in $\mathrm{pH} 6.5$ buffered water.

Experiment 10

Irradiation of a solution of mesotrione $(0.1 \mu \mathrm{M})$ in $\mathrm{pH} 6.5$ buffered water with $5 \mathrm{mg} / \mathrm{L} \mathrm{SRMON}$ Experiment 11

Irradiation of a solution of mesotrione $(0.1 \mu \mathrm{M})$ in $\mathrm{pH} 6.5$ buffered water with $15 \mathrm{mg} / \mathrm{L}$ SRMON Experiment 12

Irradiation of a solution of mesotrione $(0.1 \mu \mathrm{M})$ in $\mathrm{pH} 6.5$ buffered water with $30 \mathrm{mg} / \mathrm{L}$ SRMON

\begin{tabular}{|c|c|c|c|c|c|c|}
\hline & $\begin{array}{c}\text { Irradiation } \\
\text { time }\end{array}$ & to & $6 \mathrm{~h}$ & $12 \mathrm{~h}$ & $18 \mathrm{~h}$ & $24 \mathrm{~h}$ \\
\hline 9 & Concentration $* 10^{8}$ & 10 & 9.9 & 9.8 & 9.8 & 9.7 \\
\hline
\end{tabular}




\begin{tabular}{|c|c|c|c|c|c|c|}
\hline & $\begin{array}{c}\mathrm{mol} / \mathrm{L} \\
\pm 0.1 * 10^{8} \mathrm{~mol} / \mathrm{L}\end{array}$ & & & & & \\
\hline 10 & $\begin{array}{c}\text { Concentration } * 10^{8} \\
\mathrm{~mol} / \mathrm{L} \\
\pm 0.1 * 10^{8} \mathrm{~mol} / \mathrm{L}\end{array}$ & 10 & 10 & 10 & 9.9 & 10 \\
\hline 11 & $\begin{array}{c}\text { Concentration } * 10^{8} \\
\mathrm{~mol} / \mathrm{L} \\
\pm 0.1 * 10^{8} \mathrm{~mol} / \mathrm{L}\end{array}$ & 10 & 9.8 & 9.7 & 9.3 & 8.9 \\
\hline 12 & $\begin{array}{c}\text { Concentration } * 10^{8} \\
\mathrm{~mol} / \mathrm{L} \\
\pm 0.1 * 10^{8} \mathrm{~mol} / \mathrm{L}\end{array}$ & 10 & 8.9 & 8.6 & & 7.3 \\
\hline
\end{tabular}

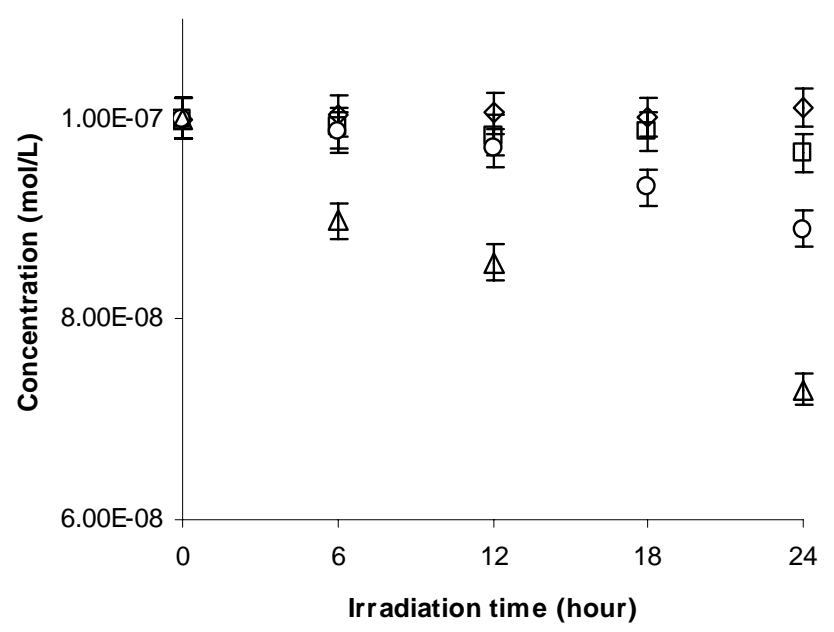

Concentration decay versus time for mesotrione $(0.1 \mu \mathrm{M})$ in $\mathrm{pH} 6.5$ buffered water $(\square)$ with no SRMON $(\diamond)$ with $5 \mathrm{mg} / \mathrm{L}$ SRMON, (O) with $15 \mathrm{mg} / \mathrm{L}$ SRMON and $(\Delta)$ with $30 \mathrm{mg} / \mathrm{L} \mathrm{SRMON}$

Experiment 13

Irradiation of a solution of mesotrione $(0.1 \mu \mathrm{M})$ in $\mathrm{pH} 6.5$ buffered water. with $50 \mu \mathrm{M}$ nitrate Experiment 14

Irradiation of a solution of mesotrione $(0.1 \mu \mathrm{M})$ in $\mathrm{pH} 6.5$ buffered water with $50 \mu \mathrm{M}$ nitrate and $30 \mathrm{mg} / \mathrm{L} \mathrm{MON}$

\begin{tabular}{|c|c|c|c|c|c|c|}
\hline & $\begin{array}{c}\text { Irradiation } \\
\text { time }\end{array}$ & to & $6 \mathrm{~h}$ & $12 \mathrm{~h}$ & $18 \mathrm{~h}$ & $24 \mathrm{~h}$ \\
\hline 13 & $\begin{array}{c}\text { Concentration } * 10^{8} \\
\mathrm{~mol} / \mathrm{L} \\
\pm 0.1 * 10^{8} \mathrm{~mol} / \mathrm{L}\end{array}$ & 10 & 9.1 & 8.4 & 8.1 & 7.3 \\
\hline 14 & $\begin{array}{c}\text { Concentration } * 10^{8} \\
\mathrm{~mol} / \mathrm{L} \\
\pm 0.1 * 10^{8} \mathrm{~mol} / \mathrm{L}\end{array}$ & 10 & 8.9 & 8.1 & 7.9 & 7.5 \\
\hline
\end{tabular}




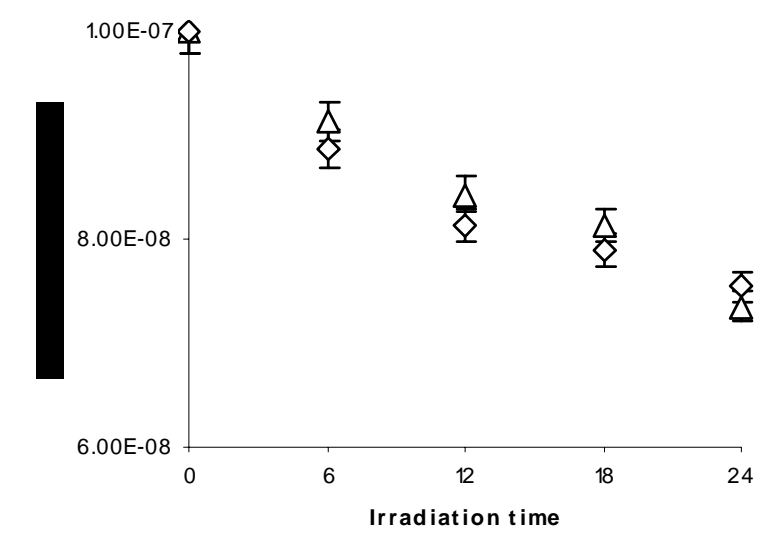

Concentration decay versus time for mesotrione $(0.1 \mu \mathrm{M})$ in $\mathrm{pH} 6.5$ buffered water $(\Delta)$ with $50 \mu \mathrm{M}$ nitrate, $(\diamond)$ with $50 \mu \mathrm{M}$ nitrate and $30 \mathrm{mg} / \mathrm{L} \mathrm{SRMON}$

\section{Experiment 15}

Irradiation of a solution of FFA $(0.1 \mathrm{mM})$ in $\mathrm{pH} 6.5$ buffered water with $30 \mathrm{mg} / \mathrm{L}$ SRMON

\begin{tabular}{|c|c|c|c|c|c|c|c|}
\hline & $\begin{array}{c}\text { Irradiation } \\
\text { Time (min) }\end{array}$ & to & 30 & 60 & 90 & 120 & 160 \\
\hline 15 & $\begin{array}{c}\text { Concentration } * 10^{5} \\
\mathrm{~mol} / \mathrm{L} \\
\pm 0.1 * 10^{5} \mathrm{~mol} / \mathrm{L}\end{array}$ & 10 & 8.1 & 6.9 & 5.7 & 5.2 & 4.4 \\
\hline
\end{tabular}

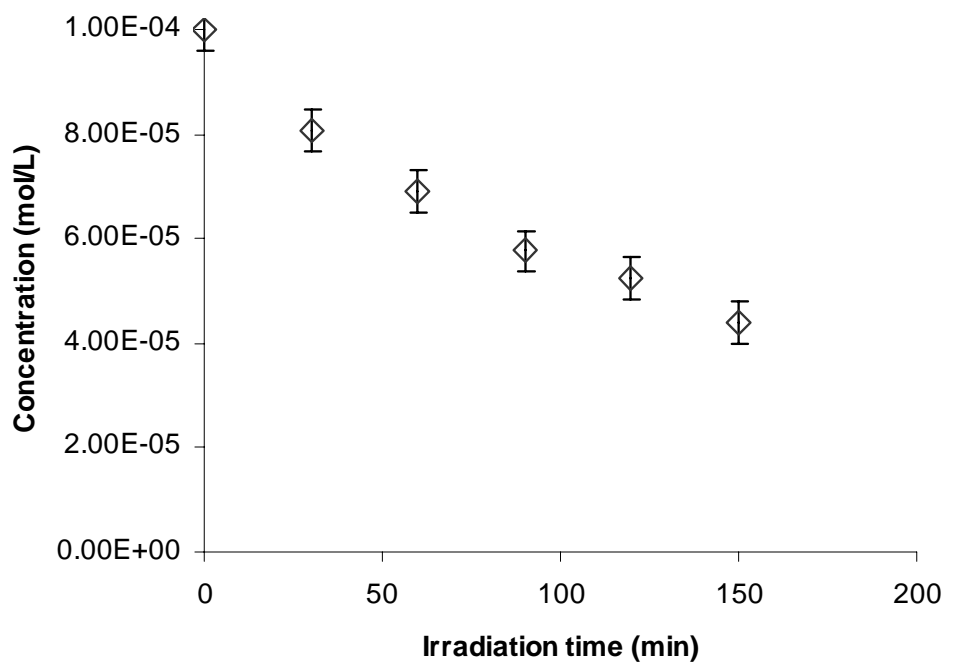

